(C) 2019 IEEE. Personal use of this material is permitted. Permission from IEEE must be obtained for all other uses, in any current or future media, including reprinting/republishing this material for advertising or promotional purposes, creating new collective works, for resale or redistribution to servers or lists, or reuse of any copyrighted component of this work in other works. 


\section{Improve Indoor Positioning Accuracy Using Filtered RSSI and Beacon Weight Approach in iBeacon Network}

\author{
Laial Alsmadi \\ Faculty of Engineering and IT \\ University of Technology Sydney \\ Sydney, Australia
}

\author{
Xiaoying Kong \\ Faculty of Engineering and IT \\ University of Technology Sydney \\ Sydney, Australia
}

\author{
Kumbesan Sandrasegaran \\ Faculty of Engineering and IT \\ University of Technology Sydney \\ Sydney, Australia
}

\begin{abstract}
Increasing the location accuracy of the Indoor Positioning System (IPS) is an important research area in localization. Utilizing mobile beacons in IPS environment has made localization more accurate and cost-effective. This paper proposes an Filtered RSSI and Beacon Weight Approach (FRBW) based on improved Received Signal Strength Indicator (RSSI) values using a Kalman filter. This algorithm takes both the distance and improved RSSI measurements between beacon nodes into consideration. Kalman filter applied on the RSSI measurements that eliminate noise of the signal and then applied on FRBW positioning algorithm. The proposed algorithm was applied using eight beacons. The results show that this FRBW approach has better positioning accuracy and minimum location error, and can be applied in IoT applications in smart city.
\end{abstract}

Keywords-Indoor positioning, IPS, beacon, Bluetooth Low Energy, Kalman filter, FRBW, IoT.

\section{INTRODUCTION}

The positioning systems are used to locate any wanted object regardless of its current location, there are many positioning systems, however, the most common and known positioning system is the Global Positioning System that can detects locations of objects in an outdoor environment [1]. Due to the signal problems with the GPS, the ability to find objects in indoor environment is limited, hence the Indoor Positioning System which is a relatively new technology that can find exact location of any object where GPS signal is lost or blocked i.e. inside the buildings and tunnels $[1,2]$. Indoor positioning is one of the most important functions in smart city applications.

Indoor positioning using Bluetooth Low Energy (BLE) beacons is an emerging technology. BLE beacons have the advantages of small size, low cost and low energy consumption [3].

Positioning using beacons is based on computing the distance between positioning device and beacons. 3 distances from 3 beacons will determine one position. There is no direct distance measurement from beacon signals. The measurement of the signal power using Received Signal Strength Indicator (RSSI) is used to indirectly compute the distance. RSSI measurements and distance computing contain errors.

In literature, research efforts have been made to minimize the distance error and increase the position accuracy. The centroid localization algorithm proposed by [4] uses the beacons coordination to estimate the position of the unknown mobile position using centroid formula, however, the position accuracy using this algorithm is very low. The Weighted Centroid Localization algorithm [5] uses the weight as a factor to estimate position. The Average Weighted Based Centroid Localization (AWBCL) algorithm proposed by [6] which is based on WCL algorithm has increased the location accuracy; however the position error is still high.

In this paper, we show how the improved measurements of RSSI values using Kalman filter have significantly improved the positioning accuracy based on beacon Weight positioning algorithm.

This paper is organized in the following sections. Section II will review the related work in literature. Section III will present our positioning algorithm. Section IV will demonstrate our experiments and discuss the results. Finally Section V will conclude the paper.

\section{RELATED WORK}

Indoor positioning technologies in literature include Radio-frequency identification (RFID), WiFi, Bluetooth, ZigBee, inertial navigation, geomagnetic, computing vision, etc. The RFID technology uses radio waves to identify and track objects automatically [7]. RFID systems consist of two elements: the transponder and the readers [8]. Wi-Fi positioning uses devices of radio wireless local area networking based on the IEEE 802.11 [9]. Using the existing Wi-Fi network for indoor positioning can minimize the cost of deployment. There is no need for extra software or hardware [10]. The Bluetooth [3] is a personal area network standard that is widely used for short distance communications. Bluetooth is easy to deploy, requires low power consumption and cheap [11].

The positioning mechanisms include time of arrival (TOA) method that depends on precise measurement of arrival time, time difference of arrival (TDOA) method that measures the relative time at each node, the angle of arrival (AOA) that measures the angle of the received signal, and the received signal strength indication (RSSI) which calculates the position based on the signal strength.

iBeacon is a new technology using Bluetooth Low Energy that was developed by Apple in 2013 [12]. The modern smartphones are equipped with BLE technology which can be utilized for indoor positioning based on their RSSI values [13],[14]. The advertisement packet that sent by iBeacon contains information such as broadcasting power, advertising interval, measured power and RSSI values [15]. The RSSI value which represents the measurement of the power present in received radio signal in $\mathrm{dBm}$ is represented 
in a negative form, therefore, the signal with -70 RSSI value is weaker than the signal with -10 RSSI values [16].

There is a very important relationship between the distance and the RSSI value. This relationship can be modeled using the Path Loss Exponent Model as explained in the next section. Using iBeacon to build an indoor positioning network is a new challenge to meet the indoor accuracy and reliability requirements in smart city applications.

The comparison of major current used indoor positioning technologies are demonstrated by [17] as the table below:

TABLE I. COMPARISON OF INDOOR POSITIONING TECHNOLOGIES [17]

\begin{tabular}{|l|c|c|c|}
\hline IPS Technology & Accuracy & Strengths & Weaknesses \\
\hline Wi-Fi & $1.5 \mathrm{~m}$ & $\begin{array}{c}\text { Low cost, good } \\
\text { precision }\end{array}$ & $\begin{array}{c}\text { Vulnerable to } \\
\text { access point } \\
\text { changes }\end{array}$ \\
\hline Bluetooth & $\begin{array}{c}30 \mathrm{~cm}- \\
\text { meters }\end{array}$ & $\begin{array}{c}\text { Low cost, good } \\
\text { precision }\end{array}$ & $\begin{array}{c}\text { Intrusive; } \\
\text { needs signal } \\
\text { mapping }\end{array}$ \\
\hline RFID & $1-5 \mathrm{~m}$ & $\begin{array}{c}\text { Very low cost } \\
\text { passive side }\end{array}$ & $\begin{array}{c}\text { Very low } \\
\text { precision }\end{array}$ \\
\hline
\end{tabular}

Many algorithms were proposed to estimate the position of the objects in indoor environment using RSSI approach. These include least square method [18], fingerprint and the weight centroid localization (WCL). The least square method uses the distance of the receiver to multi beacons to compute the position of the receiver. The accuracy of the fingerprint approach is very high but the offline phase is time consuming and expensive $[19,20]$.

The WCL proposed by [5] is a fast and simple algorithm that uses centroid algorithm to compute location of devices; the localization is computed by taking the average value of known iBeacons coordinates. Research in [21,22] proposed improvements based on WCL algorithm; however the estimated error is still significantly high.

The Averaged Weighted Based Centroid Localization proposed by [6] uses weights that are dependent on the average value of the estimated location of the estimated mobile position and the actual mobile position. AWBCL has better positioning accuracy and reduced location error than the conventional simple WCL algorithm.

To further improve the positioning accuracy and reliability, we developed a new approach using smoothed RSSI with Kalman filter and Beacon Weight positioning algorithm. This approach is presented in the following sections.

\section{INDOOR POSITIONING USING KALMAN FILTER AND BEACON WEIGHT POSITIONING}

Our indoor positioning process is developed as the following major phases: Reducing measurement errors in RSSI using Kalman filter; Computing position using Beacon Weight algorithm. The approach is designed as Figure 1. Each phase is presented in this section below.



Fig. 1. Positioning algorithm using Kalman filter and integrated beacon weight

\section{A. Filtering RSSI measurements using Kalman filter}

The received RSSI measurements have high levels of noise. Therefore, to get better and precise information, the raw RSSI measurements need filtering. In literature, researchers applied Kalman filter to estimate RSSI errors in wireless LAN based positioning [23].

In our approach for iBeacon positioning, we use Kalman filter to reduce RSSI errors. The Kalman filter is composed of a process model, and a measurement model. The process model presents the relationship among state of interest and process noises. The measurement model presents the relationship among state of interest and measurement. In our approach, the state of interest is the predicted RSSI. The measurement is the RSSI value. Their relationships are presented over time steps.

The Kalman filter for RSSI estimate is designed as follows.

State of interest $\mathrm{x}$ is designed to be RSSI at time step $\mathrm{t}$ :

$$
x_{t}=\operatorname{RSSI}(t)
$$

The process model of the Kalman filter is designed as:

$$
\dot{x}_{t}=A x_{t}+\mathcal{E}
$$

where $A=1$ in our design, $\mathcal{E}$ is the process noise.

The measurement model is designed using the relationship between the state of interest and the received RSSI measurement:

$$
Z_{t}=H x_{t}+\Gamma
$$

where $Z_{t}$ is the RSSI measurement at time step $\mathrm{t} . \mathrm{H}=1$ in our design. $\Gamma$ is the measurement noise.

We use a time step from $\mathrm{t}-1$ to $\mathrm{t}$ for Kalman filter update, and update the Kalman filter process for state of interest, Kalman gain, and variance from time step $\mathrm{t}-1$ to $\mathrm{t}$ as follows.

$$
\begin{aligned}
& \hat{\mathrm{x}}_{\mathrm{t}}^{-}=\hat{\mathrm{x}}_{\mathrm{t}-1} \\
& \overline{\mathrm{P}}_{\mathrm{t}}=\mathrm{P}_{\mathrm{t}-1}+\mathrm{Q}
\end{aligned}
$$




$$
\begin{aligned}
& \mathrm{K}_{\mathrm{t}}=\mathrm{P}_{\mathrm{t}}^{-}\left(\mathrm{P}_{\mathrm{t}}^{-}+\mathrm{R}\right)^{-1}=\frac{\mathrm{P}_{\mathrm{t}}^{-}}{\left(\mathrm{P}_{\mathrm{t}}^{-}+\mathrm{R}\right)} \\
& \hat{\mathrm{x}}_{\mathrm{t}}=\hat{\mathrm{x}}_{\mathrm{t}}^{-}+\mathrm{K}_{\mathrm{t}}\left(\mathrm{z}_{\mathrm{t}}-\hat{\mathrm{x}}_{\mathrm{t}}\right) \\
& \mathrm{P}_{\mathrm{t}}=\left(1-\mathrm{K}_{\mathrm{t}}\right) \mathrm{P}_{\mathrm{t}}^{-}
\end{aligned}
$$

where

$\hat{\mathrm{x}}_{\mathrm{t}}^{-}$is the predicted state at time step $\mathrm{t}$.

$\hat{\mathrm{x}}_{\mathrm{t}-1}$ is the state estimate at time step $\mathrm{t}-1$.

$\mathrm{Q}$ is the covariance of the process noise.

$\mathrm{R}$ is the covariance of measurement noise.

$\mathrm{P}_{\mathrm{t}}^{-}$is the predicted error variance.

$P_{t}$ is the updated error variance.

$\mathrm{K}_{\mathrm{t}}$ is the Kalman gain at time step $\mathrm{t}$.

The distance between the iBeacon and mobile device is calculated using the Path Loss Model with the relationship between RSSI and distance [24, 25]:

$$
\text { RSSI_KF }=\operatorname{nlog} 10^{\left(\frac{\mathrm{d} i j}{d 0}\right)}+A 0
$$

where RSSI KF is the improved received value in $\mathrm{dBm}$ at distance dij using Kalman filter. A0 is the received RSSI at one meter, $\mathrm{d} 0$ is the reference distance and $\mathrm{n}$ is the path-loss exponent and $\mathrm{d}$ is the measured distance after applying Kalman filter and is calculated using Equation 10.

$\mathrm{d}_{\mathrm{i}}=10^{\left({ }^{\left.\left(\text {TxPower }-R S S I_{-} K F\right) / 2 n\right)} \quad(\mathrm{i}=1,2 . . \mathrm{n})\right.}$

In our experiment process, the indoor movement is assumed to happen in the same level of a building. We use $2 \mathrm{D}$ only for positioning.

Assume there are $\mathrm{n}$ beacon nodes and the location of the mobile position is $\left(\mathrm{x}_{\mathrm{o}}, \mathrm{y}_{\mathrm{o}}\right), \mathrm{d}_{\text {true }_{\mathrm{i}}}$ is the estimated distance between the smartphone node and the ith beacon node $\left(\mathrm{x}_{\mathrm{i}}, \mathrm{y}_{\mathrm{i}}\right)$ obtained by using the log-model, while $\mathrm{d}_{\text {true } \mathrm{i}}$ represents the true distance.

$\mathrm{d}_{\text {true } \mathrm{i}}=\sqrt{\left(\mathrm{x}_{\mathrm{i}}-\mathrm{x}_{\mathrm{o}}\right)^{2}+\left(\mathrm{y}_{\mathrm{i}}-\mathrm{y}_{\mathrm{o}}\right)^{2}} \quad(\mathrm{i}=1,2 . \mathrm{n})$

The error in distance is calculated using Equation 12.

$$
\Delta \mathrm{d}_{\mathrm{i}}=\mathrm{d}_{\mathrm{i}}-\mathrm{d}_{\text {true_i }} \quad(\mathrm{i}=1,2, \ldots \mathrm{n})
$$

$\Delta \mathrm{d}_{\mathrm{i}}$ is the distance error from the receiver to the ith beacon.

As we have deployed eight beacons, there will be eight distance error; the least three distance error will be used to find the estimated mobile position using our proposed approach as explained in the next section.

\section{B. Computing Positioning Using Beacon Weight}

The AWBCL algorithm proposed by previous researchers is based on WCL technique that assign weight (wi) to each beacon based on distance to beacon that we found using improved RSSI values using Kalman filter and degree $(\mathrm{g})$. The weight is calculated using Equation 13, assuming $g=1$ and dij is calculated using Equation 10:

$$
w_{i j}=\frac{1}{\left(d_{i j}\right)^{g}}
$$

The distributed Beacons position is known which is $\mathrm{Bi}$ (xi, yi), where $1<=\mathrm{i}<=\mathrm{n}$, as we have distributed eight beacons in our experiment, then $n=8$. The estimated position of the unknown mobile Mob $\left(\mathrm{X}_{\text {est }}, \mathrm{Y}_{\mathrm{est}}\right)$ is calculated based on the integrated weight.

Wight of a point of intersection of Beacon 1 and Beacon 2 is the integrated weights of Beacon 1 and Beacon 2 as following:

$$
\begin{aligned}
& \mathrm{w} 1_{\mathrm{avg}}=\mathrm{w} 1+\mathrm{w} 2 \\
& \mathrm{w} 2_{\mathrm{avg}}=\mathrm{w} 1+\mathrm{w} 3 \\
& \mathrm{w} 3_{\mathrm{avg}}=\mathrm{w} 2+\mathrm{w} 3
\end{aligned}
$$

In our approach, there are six intersection points, $\left(\mathrm{XC}_{\mathrm{ac}}, \mathrm{YC}_{\mathrm{ac}}\right)$ is the point intersection between Beacon 1 and Beacon $3,\left(\mathrm{XC}_{\mathrm{ab}}, \mathrm{YC}_{\mathrm{ab}}\right)$ is the point intersection between Beacon 1 and Beacon $2,\left(\mathrm{XC}_{\mathrm{bc}}, \mathrm{YC}_{\mathrm{bc}}\right)$ is the point intersection between Beacon 2 and Beacon 3 , where weight w is calculated using Equation 13.

$$
\begin{aligned}
& X_{e s t}=\frac{w 1_{a v g} * X C_{a c}+w 2_{a v g} * X C_{a b}+w 3_{a v g} * X C_{b c}}{\sum_{i=1}^{n} w i_{a v g}} \\
& Y_{e s t}=\frac{w 1_{a v g} * Y C_{a c}+w 2_{a v g} * Y C_{a b}+w 3_{a v g} * Y C_{b c}}{\sum_{i=1}^{n} w i_{a v g}}
\end{aligned}
$$

This FRBW algorithm is validated using experiment and the results will be discussed in the next section.

\section{FRBW ALGORITHM VALIDATION USING IBEACON EXPERIMENT}

To validate the our indoor positioning approach, we use real iBeacon sensors from estimote and smart phone in an indoor environment. The mobile was tested on eight different locations and the calculated estimated mobile position were compared with the actual mobile position.

\section{A. Experiment Design}

Eight iBeacons with known coordination named $\mathrm{B} 1(\mathrm{x} 1, \mathrm{y} 1), \mathrm{B} 2(\mathrm{x} 2, \mathrm{y} 2), \ldots \mathrm{Bi}(\mathrm{xi}, \mathrm{yi})(\mathrm{i}=1,2, \ldots 8)$ were deployed in the experiment area. The mobile device was positioned in twenty different locations to validate our approach. The iBeacons RSSI values were collected using smart phone application. The collected RSSI values were then smoothed using Kalman filter. The weight of each beacon then is calculated and applied to mobile position estimation. Figure 2 shows the deployed iBeacons position and the twenty different mobile locations. 


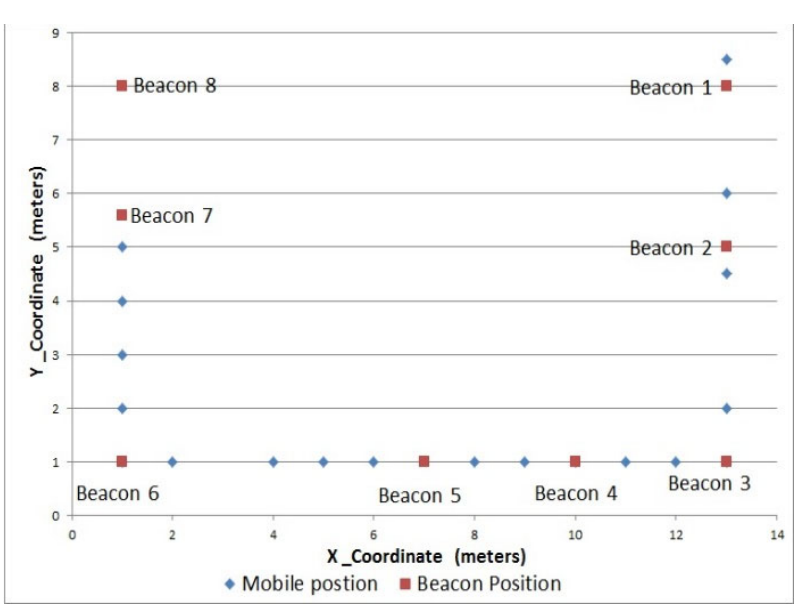

Fig.2. Experiment setup - Eight beacons and twenty moible positioins

The following table shows the experiments condition and algorithm parameters.

TABLE II. EXPERIMENT PARAMETERS

\begin{tabular}{|l|c|}
\hline \multicolumn{1}{|c|}{ Parameters } & Value \\
\hline Number of iBeacons & 8 \\
\hline $\begin{array}{l}\text { iBeacons Z coordinate } \\
\text { (beacon height) }\end{array}$ & $210 \mathrm{~cm}$ \\
\hline Receiver & Android mobile \\
\hline Filtering approach & Kalman Filter \\
\hline Path loss exponent (n) & 2 \\
\hline
\end{tabular}

\section{B. Reduced RSSI Measurement Error Using Kalman Filter}

The raw RSSI measurements contain a lot of noise that affect on the estimated mobile position. We applied Kaman filter on all received RSSI values to eliminate the noise and smooth the signal. Figure 3 shows the raw RSSI measurements, and processed RSSI values after applying Kalman filter. The results show that the noises are largely reduced.

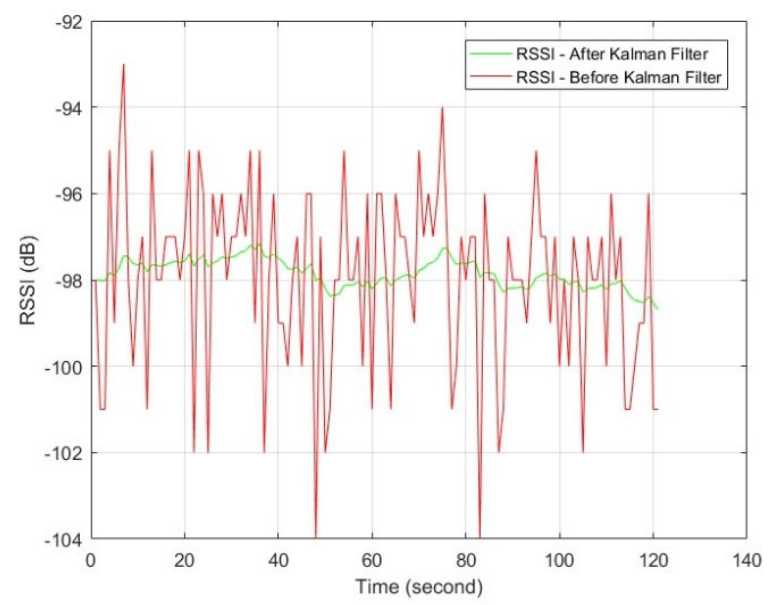

Fig. 3. Raw RSSI measurements and processed RSSI signals after applying Kalman filter

\section{Beacon Weight}

The weight of each beacon was calculated using Equation 13. Figure 4 shows the weight of Beacons 1, 2 and 3.

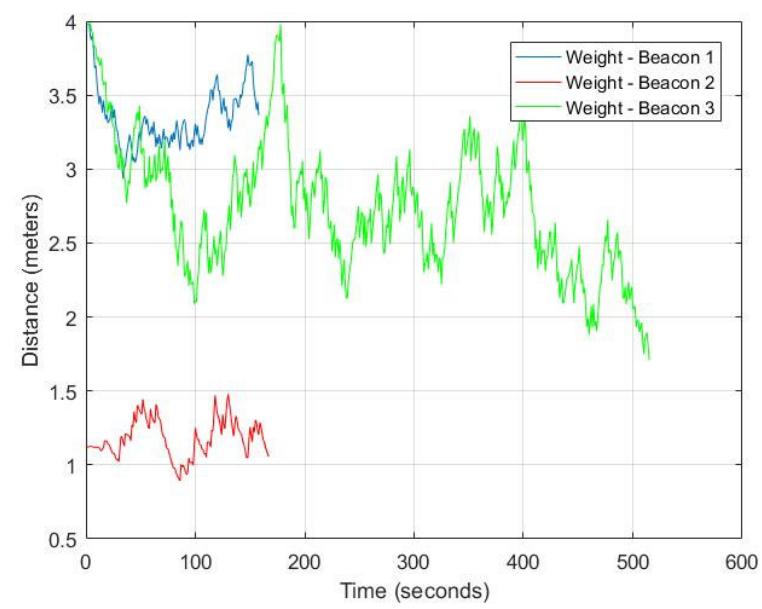

Fig. 4. Beacon weight

\section{Positioning Results Using Filtered RSSI and Beacon Weight}

In positioning experiments, we use the following steps to estimate the smartphone position:

- Apply Kalman filter on the RSSI raw measurement to eliminate the noise using Equations 4,5,6,7,8.

- Calculate distance value using smoothed RSSI estimation using Equation 9..

- Computing the weight of each beacon using Equation 13.

- Calculate the integrated weight between the beacons using Equations 14, 15, 16.

- The estimated smartphone position is calculated using Equations 17, 18.

The experiments were conducted by deploying eight estimote beacons that transmit advertisement packets periodically and an Android mobile device that receives the advertisement. The beacons' locations were predefined and fixed, the RSSI values were measured and recorded for one hour at twenty different locations. The RSSI values were then filtered using Kalman filter and the weight for each beacon were then calculated. The proposed algorithm was then applied. Figure 5 shows the actual mobile positions and the estimated mobile positions using our developed approach. 


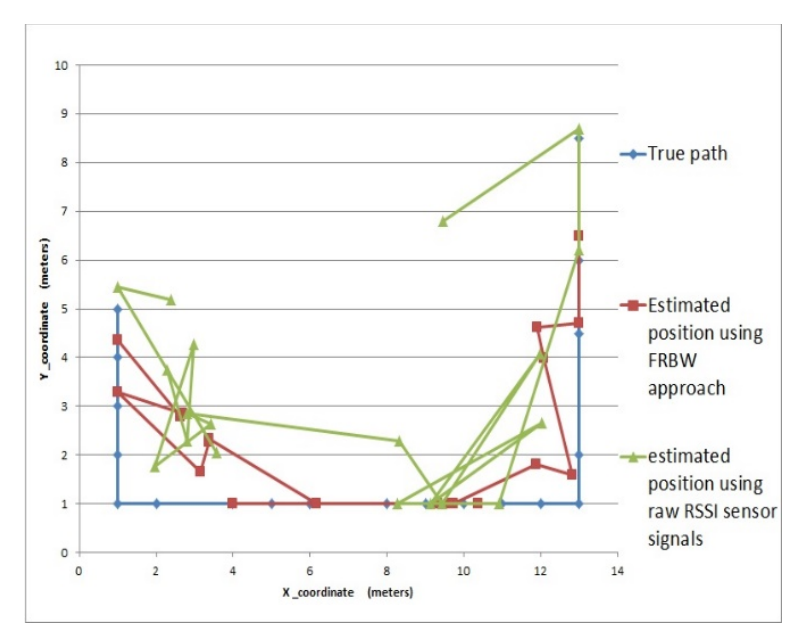

Fig. 5. Positioning results using 8 beacons

In Figure 5, the estimated positions have large errors without using RSSI filtering and beacon weight. Using our FRBW approach, the indoor positioning accuracy is improved. This level of accuracy can be used in applications such as museum navigation, airport navigation, hospital navigation, shopping mall navigation, multi-level building fire exit finding, car park navigation, and other location based services in IoT applications.

\section{CONCLUSION}

This paper presents a novel indoor positioning approach FRBW using two phases: filtering RSSI measurements and estimating position using beacon weight. This approach is validated using iBeacon network. The accuracy level is improved and can be applied in indoor systems such as airport navigation, car park navigation systems.

\section{REFERENCES}

[1] Wikipedia - GPS. [Online]. Available: https://en.wikipedia.org/wiki/Global_Positioning_System.[Accessed: 15- Oct- 2018]

[2] GPS. GPS Accuracy. [Online] Available: https://www.gps.gov/systems/gps/performance/accuracy/ . [Accessed: 15- Oct- 2018]

[3] Bluetooh. Bluetooth Specifications. [Online]. Available: https://www.bluetooth.com/ . [Accessed: 20- Oct- 2018]

[4] N. Bulusu, J. Heidemann, D. Estrin, "GPS-less low cost outdoor localization for very small devices", IEEE Personal Communications Magazine, vol. 7, no. 5, pp. 28-34, October 2000.

[5] J. Blumenthal, R. Grossmann, F. Golatowski, and D. Timmermann, "Weighted centroid localization in zigbee-based sensor networks," in Intelligent Signal Processing, 2007. WISP 2007. IEEE International Symposium on, 2007, pp. 1-6.

[6] M. ARUN, N. SIVASANKARI, P. VANATHI, and P. MANIMEGALAI, "Analysis of Average Weight Based Centroid Localization Algorithm for Mobile Wireless Sensor Networks."
[7] WiKi - RFID. Available: https://en.wikipedia.org/wiki/Radiofrequency_identification

[8] K. Finkenzeller and R. Waddington, RFID Handbook: Radiofrequency identification fundamentals and applications: Wiley New York, 1999.

[9] wikipedia - wifi. Available: https://en.wikipedia.org/wiki/Wi-Fi

[10] M. B. Ismail, A. Fathi, A. Boud, W. Nurdiana, and W. Ibrahim, "Implementation of location determination in a wireless local area network (WLAN) environment," in Advanced Communication Technology, 2008. ICACT 2008. 10th International Conference on, 2008, pp. 894-899.

[11] S. Zhou and J. K. Pollard, "Position measurement using Bluetooth," IEEE Transactions on Consumer Electronics, vol. 52, pp. 555-558, 2006.

[12] Apple. (2015, 13/8). iOS: understanding iBeacon, available: "iOS: Understanding iBeacon

[13] J. Boucaron, A. Coadou, and R. de Simone, "Synthesis of embedded software: frameworks and methodologies for correctness by construction software design, Shukla, Sandeep Kumar and Talpin, Jean-Pierre," ed: Springer, Heidelberg, 2010.

[14] R. Faragher and R. Harle, "Location fingerprinting with bluetooth low energy beacons," IEEE journal on Selected Areas in Communications, vol. 33, pp. 2418-2428, 2015.

[15] N. Newman, "Apple iBeacon technology briefing," Journal of Direct, Data and Digital Marketing Practice, vol. 15, pp. 222-225, 2014.

[16] Wikipedia. RSSI. Available: [https://en.wikipedia.org/wiki/Received_signal_strength_indication

[17] R. F. Brena, J. P. García-Vázquez, C. E. Galván-Tejada, D. MuñozRodriguez, C. Vargas-Rosales, and J. Fangmeyer, "Evolution of indoor positioning technologies: A survey," Journal of Sensors, vol. 2017, 2017.

[18] Y. Wang, X. Yang, Y. Zhao, Y. Liu, and L. Cuthbert, "Bluetooth positioning using RSSI and triangulation methods," in Consumer Communications and Networking Conference (CCNC), 2013 IEEE, 2013, pp. 837-842.

[19] P. Deepesh, R. Rath, A. Tiwary, V. N. Rao, and N. Kanakalata, "Experiences with using iBeacons for Indoor Positioning," in Proceedings of the 9th India Software Engineering Conference, 2016, pp. 184-189.

[20] L. Chen, B. Li, K. Zhao, C. Rizos, and Z. Zheng, "An improved algorithm to generate a Wi-Fi fingerprint database for indoor positioning," Sensors, vol. 13, pp. 11085-11096, 2013.

[21] Y. Zhao, X. Li, Y. Wang, and C.-Z. Xu, "Biased Constrained Hybrid Kalman Filter for Range-Based Indoor Localization," IEEE Sensors Journal, vol. 18, pp. 1647-1655, 2018 .

[22] M. Ji, J. Kim, Y. Cho, Y. Lee, and S. Park, "A novel Wi-Fi AP localization method using Monte Carlo path-loss model fitting simulation," in Personal Indoor and Mobile Radio Communications (PIMRC), 2013 IEEE 24th International Symposium on, 2013, pp. 3487-3491.

[23] V. Apte and Y. A. Powar, "Improving the accuracy of wireless LAN based location determination systems using Kalman filter and multiple observers," in Wireless Communications and Networking Conference, 2006. WCNC 2006. IEEE, 2006, pp. 463-468.

[24] Chen, Z., Zhu, Q., Jiang, H. and Soh, Y.C., "Indoor localization using smartphone sensors and iBeacons". In 2015 IEEE 10th Conference on Industrial Electronics and Applications (ICIEA) pp. 1723-1728.

[25] Tseng, C.H. and Yen, J.S., Enhanced Gaussian mixture model of RSSI purification for indoor positioning. Journal of Systems Architecture, 81, pp.1-6. 2017 